\title{
Determination of the critical Shields number for particle erosion in laminar flow
}

\author{
Malika Ouriemi, Pascale Aussillous, and Marc Medale \\ IUSTI-CNRS UMR 6595, Polytech'Marseille, Technopôle de Château-Gombert, \\ 13453 Marseille Cedex 13, France \\ Yannick Peysson \\ Institut Français du Pétrole, 1-4 avenue de Bois-Préau, 92852 Rueil-Malmaison Cedex, France \\ Élisabeth Guazzelli \\ IUSTI-CNRS UMR 6595, Polytech'Marseille, Technopôle de Château-Gombert, \\ 13453 Marseille Cedex 13, France
}

(Received 20 February 2007; accepted 8 May 2007; published online 28 June 2007)

\begin{abstract}
We present reproducible experimental measurements for the onset of grain motion in laminar flow and find a constant critical Shields number for particle erosion, i.e., $\theta^{c}=0.12 \pm 0.03$, over a large range of small particle Reynolds number: $1.5 \times 10^{-5} \leqslant \operatorname{Re}_{p} \leqslant 0.76$. Comparison with previous studies found in the literature is provided. (C) 2007 American Institute of Physics.
\end{abstract}

[DOI: 10.1063/1.2747677]

Erosion of particles by shearing flows commonly occurs in a wide variety of natural phenomena, such as sediment transport or dune formation, and of industrial processes, such as hydrate or sand issues in oil production and granular flow in food or pharmaceutical industries. The traditional way of representing the incipient motion of the grains is to use the Shields curve, which relates the dimensionless critical shear stress to the Reynolds number of the flow. ${ }^{1,2}$ This dimensionless critical shear stress, also called the Shields number, is constructed as the ratio between the shear stress at the top of the particle bed and the apparent weight of a single particle.

Most of the data are available in the turbulent regime and present large scatters due to systematic methodological biases of incipient motion of the bed. ${ }^{1-4}$ Values determined from bedload transport rate are usually larger than those deduced from visual observation of the grain motion. The discrepancy between the experiments may also be due to differences between the initial state of the bed as erosion and deposition are very sensitive to bed packing conditions. ${ }^{5}$ The presumably simpler case of laminar flow suffers from the same difficulty. ${ }^{6-9}$ The scatter of the data is also due to the multiple possible definition for incipient motion. The objective of this work is to provide a robust and reproducible experimental measurement for the onset of grain motion in laminar flow and infer the critical Shields number for particle erosion.

Four different batches of spheres [polystyrene particles supplied by Maxi-Blast, polymethylmethacrylate (PMMA) particles by Lehmann \& Voss \& Co., and glass particles by Potters-Ballotini] were used to perform the experiments. The particle size distributions were measured with a digital imaging system. The particle diameter distributions were observed to be approximately Gaussian for all the different batches and were therefore well represented by a mean diameter $d$ indicated in Table I (the error corresponds to one standard deviation). The particle density $\rho_{p}$ (also listed in
Table I) was determined using a pycnometer. Experiments were carried out using four different mixtures of distilled water and UCON oil 75H-90000 supplied by Chempoint. The viscosity $\eta$ and the density $\rho_{f}$ of these different mixtures are listed in Table II.

The experimental test section was a horizontal glass tube of length $L=1.8 \mathrm{~m}$ and inner diameter $D=3 \mathrm{~cm}$. First, the tube was filled with fluid and the particles were carefully introduced to build an uniform flat bed. Second, a constant flow rate was imposed. The pipe flow was driven by gravity using continuous overflow from an overhead tank, the elevation of which was varied. At the outlet from the test section, the particles were captured by a mesh while the fluid was run into a thermostated fluid reservoir. From this lower reservoir, the fluid was continuously returned to the overflowing reservoir by a pump. This arrangement isolated the test section from the pump and insured a constant temperature $T$ across the whole experimental loop. Note that the captured particles were not re-injected into the test section.

For a given flow rate and combination of fluid and particles, the initial height of the bed $h_{p}^{\text {start }}$ was varied to fill up $15 \%$ to $85 \%$ of the tube diameter $D$. The evolution of the bed height was then recorded as a function of time. The bed was illuminated by a laser sheet positioned perpendicularly to its surface and aligned with the tube length in its middle. The illuminated upper layer of particles intersecting the sheet was imaged by a digital camera. The images were then analyzed (with ImageJ available at http://rsb.info.nih.gov/ij/) to yield the position of the fluid-particle interface. Each image was thresholded to turn this interface into a white curve that was further eroded to a single-pixel-thick curve. After calibration, this provides a precise measurement of the fluid-particle interface with an accuracy of $0.8 \mathrm{~mm}$. In order to perform the calibration, a grid was inserted into the tube filled with pure fluid. An image of this grid was then recorded under the same optical conditions used in interface-position measure- 
TABLE I. Particle characteristics.

\begin{tabular}{lccc}
\hline \hline Batch & Composition & $d(\mu \mathrm{m})$ & $\rho_{p}\left(\mathrm{~g} / \mathrm{cm}^{3}\right)$ \\
\hline A $(\bigcirc)$ & Glass & $132 \pm 22$ & $2.490 \pm 0.003$ \\
B $(\square)$ & Polystyrene & $538 \pm 24$ & $1.051 \pm 0.002$ \\
C $(\triangle)$ & PMMA & $132 \pm 20$ & $1.177 \pm 0.002$ \\
D $(\diamond)$ & PMMA & $193 \pm 30$ & $1.177 \pm 0.002$ \\
\hline \hline
\end{tabular}

ments and the coordinates of its points were measured. The flow rate $Q_{\text {pipe }}$ was measured with a flowmeter with an accuracy of $3.2 \%$.

One can explore the threshold of motion by gradually increasing the flow rate until a single particle is entrained by the flow. This threshold strongly depends on the way the bed is prepared. When increasing the flow rate above this first threshold and until a second threshold is reached, the particle flux decreases and eventually vanishes with time due to the rearrangement of the particles near the bed surface. Above this second threshold, the particle flux reaches a nonzero saturated value. This saturated threshold is expected to be independent of the initial preparation of the bed. ${ }^{8}$ These two thresholds are observed in the present experiments but the value of the second threshold is not easy to capture as one needs to measure the particle flux and checks that it is indeed saturated. Here, we choose instead to characterize this second threshold through the cessation of motion. We check that this threshold corresponds indeed to the threshold of incipient motion as, by increasing the flow rate by a small amount, particles are set again in motion.

For small initial height, the bed height is not observed to change in time although rearrangement of particles can occur. The final bed height $h_{p}^{\text {end }}$ is then equal to $h_{p}^{\text {start }}$, as can be seen in Fig. 1. Above a critical initial height $h_{p}^{\text {start }} \geqslant h_{p}^{c}\left(h_{p}^{c}\right.$ $=0.5 \mathrm{D}$ in Fig. 1), the bed shape evolves and can either becomes slightly tilted or forms dunes moving downward. Since the test section is not fed in with particles and that a layer of particles is carried downward, the total amount of particles decreases with time. When the experiment is run for a long enough time (from two days to two weeks), the bed shape eventually freezes, exhibiting either a flat surface or dunes of uniform amplitude, as there is no particle motion and the bed compaction has reached a final state on its own. In other words, the onset for cessation of motion has been reached. The final maximum height of the bed is found to be constant, $h_{p}^{\text {end }}=h_{p}^{c}$, for $h_{p}^{\text {start }} \geqslant h_{p}^{c}$, as observed in Fig. 1 . The shear stress applied at the top of the bed surface is related to the bed height and the flow rate. As for a given flow rate the

TABLE II. Fluid characteristics.

\begin{tabular}{ccccc}
\hline \hline Fluid & $\%$ UCON & $T\left({ }^{\circ} \mathrm{C}\right)$ & $\eta(\mathrm{cP})$ & $\rho_{f}\left(\mathrm{~g} / \mathrm{cm}^{3}\right)$ \\
\hline 1 & 0 & 20 & $1.00 \pm 0.05$ & $1.004 \pm 0.001$ \\
2 & 0 & 35 & $0.70 \pm 0.04$ & $0.999 \pm 0.001$ \\
3 & 12 & 35 & $8.8 \pm 0.4$ & $1.023 \pm 0.001$ \\
4 & 20 & 35 & $40 \pm 2$ & $1.040 \pm 0.001$ \\
\hline \hline
\end{tabular}

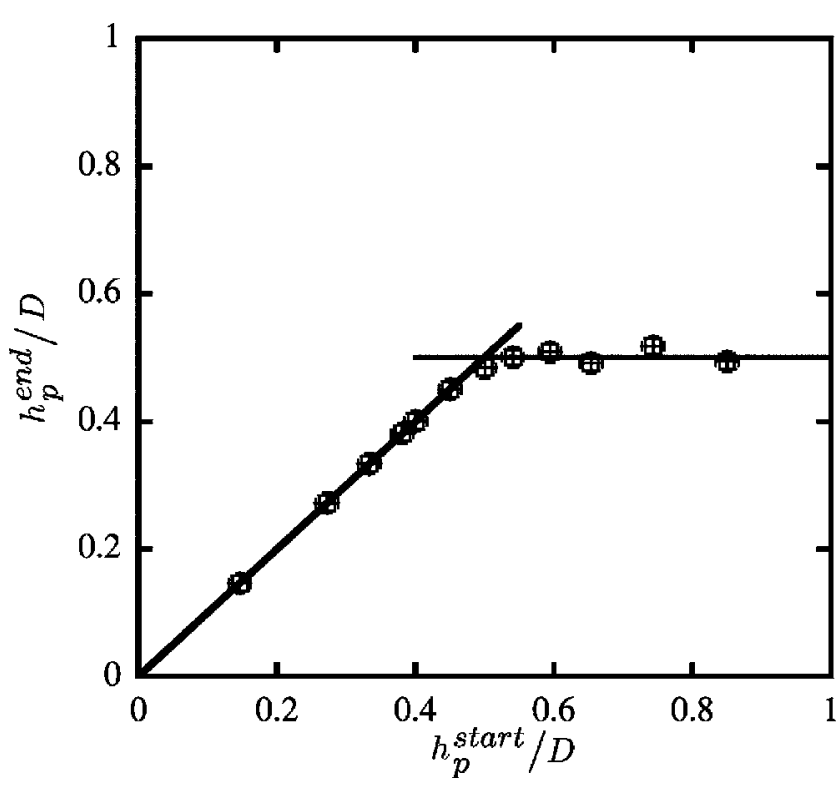

FIG. 1. Normalized final height of the bed $h_{p}^{\text {end }} / D$ vs normalized initial height of the bed $h_{p}^{\text {start }} / D$ for particles of batch $\mathrm{B}$ in fluid 2 at a flow rate of $Q_{\text {pipe }}=(2.77 \pm 0.09) \times 10^{-5} \mathrm{~m}^{3} / \mathrm{s}$. The solid lines represent the slope one (left) and the constant $h_{p}^{c}$ (right).

final height is a constant, the onset for cessation of motion corresponds to a constant critical shear stress.

Figure 2 shows $h_{p}^{c} / D$ versus the dimensionless flow rate, i.e., the pipe Reynolds number $\operatorname{Re}_{\text {pipe }}=4 \rho_{f} Q_{\text {pipe }} / \pi \eta D$, for different combinations of fluid and particles. For each curve, as the flow rate is increased, $h_{p}^{c} / D$ is seen to diminish. This behavior is again consistent with the idea of a constant critical shear stress for the onset for cessation of motion. Particles of batch A have been used in two different fluids where the viscosity was varied by a factor of 4 . For a given non-

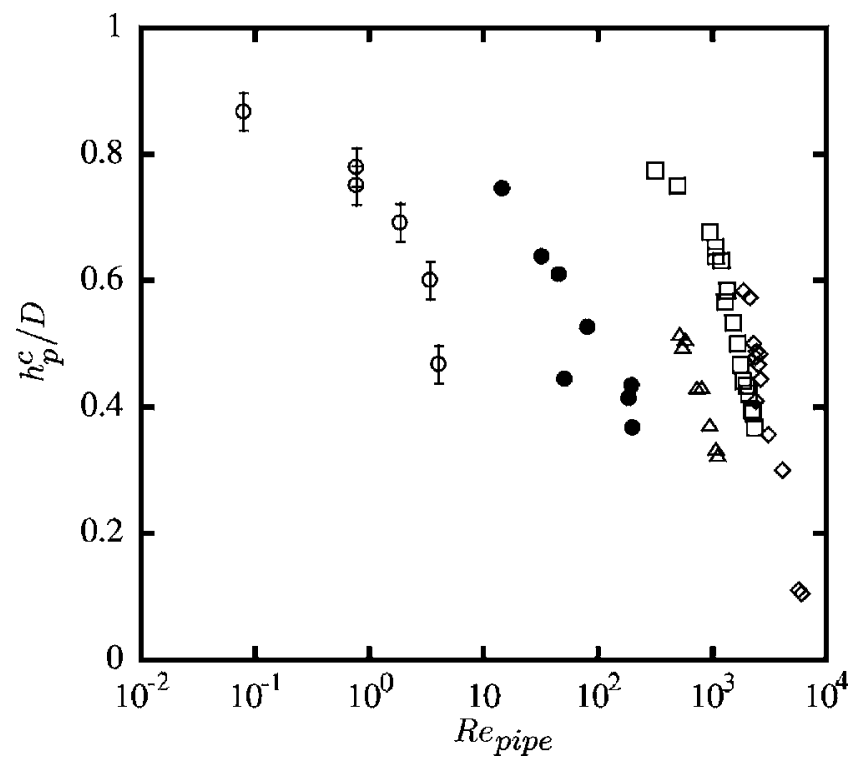

FIG. 2. Normalized critical height of the bed $h_{p}^{c} / D$ vs Reynolds number $\mathrm{Re}_{\text {pipe }}$ for batch A in fluid $3(\bullet)$, for batch A in fluid $4(\bigcirc)$, for batch B in fluid $2(\square)$, for batch $C$ in fluid $1(\triangle)$, and for batch D in fluid $2(\diamond)$. The error bars are only indicated for batch A in fluid $4(\bigcirc)$. Note that the horizontal error bar is smaller than the size of the symbol. 
normalized flow rate $Q_{\text {pipe }}$, the final height is found to be smaller for the larger viscosity. Again, this behavior could be related to the existence of a constant viscous critical shear stress. When $Q_{\text {pipe }}$ is normalized (see Fig. 2), the two sets of data $(\bigcirc$ and $\bigcirc)$, however, do not collapse into the same curve. In the same way, data coming from experiments using batches B $(\square)$ and D $(\diamond)$ in the same fluid are not completely superimposed. Therefore, the simple scaling with $\mathrm{Re}_{\text {pipe }}$ is not sufficient to obtain a collapse of the curves. The relation between $h_{p}^{c} / D$ and $\mathrm{Re}_{\text {pipe }}$ need to be interpreted in terms of the Shields number.

For simplicity, we first consider the case of a Poiseuille flow in a two-dimensional channel of thickness $D$ comprising a flat solid bed of height $h_{p}=D-h_{f}$, where $h_{f}$ is the fluid height. We take a viscous stress at the top of the bed $\tau_{2 D}$ $=\eta \dot{\gamma}_{2 D}$ with a shear rate $\dot{\gamma}_{2 D}=6\left(Q_{2 D} / D^{2}\right)\left(D / h_{f}\right)^{2}$ and a channel flow rate $Q_{2 D}$, and build the Shields number $\theta_{2 D}$ $=\eta \dot{\gamma}_{2 D} /\left(\rho_{p}-\rho_{f}\right) g d$. The Shields number is then related to the channel Reynolds number $\operatorname{Re}_{2 D}=\rho_{f} Q_{2 D} / \eta$ by the equation

$$
\operatorname{Re}_{2 D}=\frac{\theta_{2 D}}{6} \frac{\left(\rho_{p}-\rho_{f}\right) \rho_{f} g d^{3}}{\eta^{2}}\left(\frac{D}{d}\right)^{2}\left(\frac{h_{f}}{D}\right)^{2},
$$

where one recognizes the Galileo number $\mathrm{Ga}=\left(\rho_{p}\right.$ $\left.-\rho_{f}\right) \rho_{f} g d^{3} / \eta^{2}$, where $g$ is the acceleration of gravity.

We now return to the more complicated experimental geometry of a pipe partially filled by a flat solid bed. The pipe Reynolds number is now $\mathrm{Re}_{\text {pipe }}=4 \rho_{f} Q_{\text {pipe }} / \pi \eta D$. The Shields number is again $\theta_{\text {pipe }}=\eta \dot{\gamma}_{\text {pipe }} /\left(\rho_{p}-\rho_{f}\right) g d$. The shear rate $\dot{\gamma}_{\text {pipe }}$ has been numerically computed at the top of the solid bed surface, assuming a uniform cross section along the longitudinal direction. The fluid velocity has only one nonvanishing component and the incompressible Navier-Stokes equations reduce to the Stokes equations. These equations have been solved with a no-slip boundary condition along the wetted perimeter and an imposed flow rate. They are discretized in space using the standard Galerkin finite element method and piecewise biquadratic approximations on an appropriate mesh with an accuracy of $10^{-6}$ in the L2 norm. As demonstrated in Fig. 3, a similar dependence $\dot{\gamma}_{\text {pipe }}=6 k\left(Q_{\text {pipe }} / D^{3}\right)\left(D / h_{f}\right)^{2}$ is found in the limit $0.2 \leqslant h_{f} / D$ $\leqslant 0.8$ with the numerical coefficient $k=1.85 \pm 0.02$. In these limits, we found the new equation that substitutes for Eq. (1) in the case of a pipe partially filled by a solid bed:

$$
\mathrm{Re}_{\text {pipe }}=\frac{2 \theta_{\text {pipe }}}{3 k \pi} \mathrm{Ga}\left(\frac{D}{d}\right)^{2}\left(\frac{h_{f}}{D}\right)^{2} .
$$

In Fig. 4, we have plotted in logarithmic scales the data of Fig. 2 by taking $h_{f}^{c} / D=1-h_{p}^{c} / D$ instead of $h_{p}^{c} / D$ and using the dimensionless flow rate $\operatorname{Re}_{\text {pipe }}(d / D)^{2} / \mathrm{Ga}=\mathrm{Re}_{p} / \mathrm{Ga}$, where $\operatorname{Re}_{p}=\operatorname{Re}_{\text {pipe }}(d / D)^{2}$ is the particle Reynolds number. The data collapse onto a single line. The logarithm of $\mathrm{Re}_{p} / \mathrm{Ga}$ has been fitted to a linear function of the logarithm of $h_{f}^{c} / D$ by the method of weighted least squares. This gives a slope of $1.98 \pm 0.08$ in good agreement with the exponent 2 given by Eq. (2). The data outside the limit of validity of the equation have been excluded from the fitting. We can conclude that Eq. (2) is in fairly good agreement with the experimental data in the limit $0.2 \leqslant h_{f} / D \leqslant 0.8$. In this range,

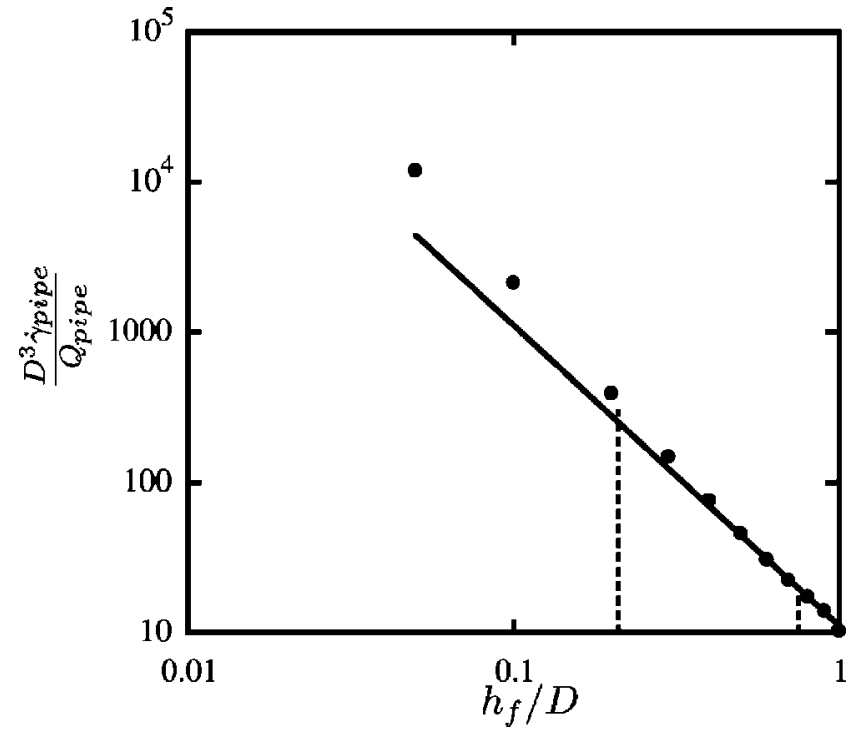

FIG. 3. Numerical dimensionless fluid shear rate $D^{3} \dot{\gamma}_{\text {pipe }} / Q_{\text {pipe }}$ at the bed surface $h_{p} / D$ vs normalized height of the fluid $h_{f} / D$. The solid line represents the slope -2 . The dotted lines indicate the limits $0.2 \leqslant h_{f} / D \leqslant 0.8$.

we can infer the critical value of the Shields number for cessation of motion by using the best fit with exponent 2 and the numerical coefficient $k=1.85 \pm 0.02$. We find $\theta^{c}$ $=0.12 \pm 0.03$ in the range $1.5 \times 10^{-5} \leqslant \operatorname{Re}_{p} \leqslant 0.76$.

Figure 5 compares our data (solid horizontal line) with previous results found in the literature. ${ }^{6-11}$ As in the previous studies the particle Reynolds number has been defined in a different way by taking the shear at the top of the bed $\dot{\gamma}_{c}$, the critical Shields number $\theta^{c}$ is plotted versus this new defined number $\dot{\gamma}_{c} d^{2} \rho_{f} / \eta$. Note that this new number is in fact $\theta^{c} / \mathrm{Ga}$ in the viscous regime. Therefore, this representation commonly found in the literature shows the variation in $\mathrm{Ga}$ and is somehow circular.

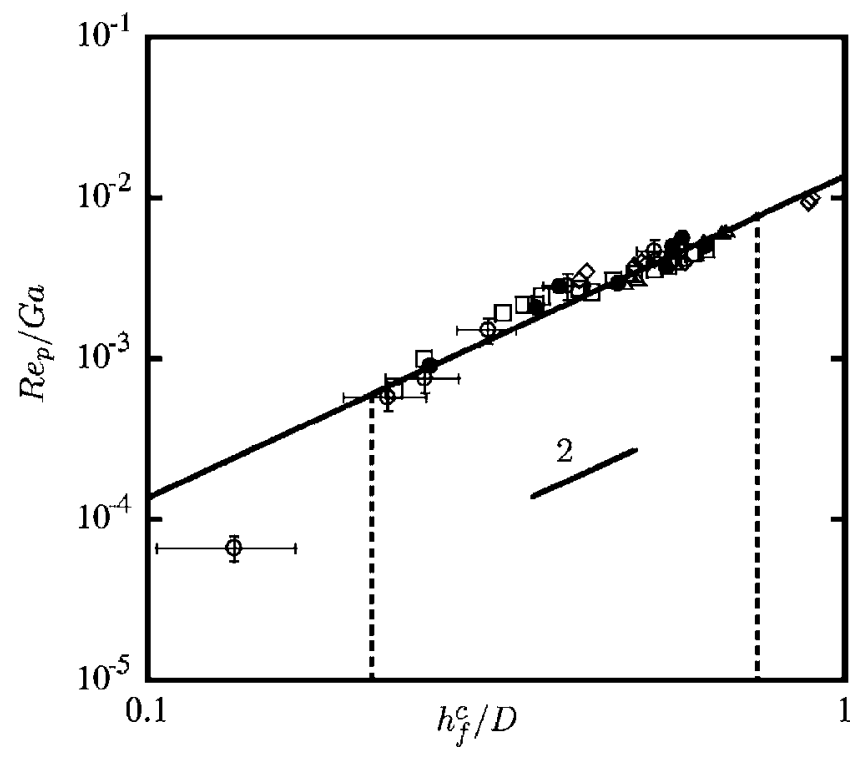

FIG. 4. Dimensionless ratio $\operatorname{Re}_{p} / \mathrm{Ga}=\operatorname{Re}_{\text {pipe }}(d / D)^{2} / \mathrm{Ga}$ vs normalized final fluid height $h_{f}^{c} / D=1-h_{p}^{c} / D$. The solid line represents the best fit. The dotted lines enclose the domain of validity of the model. 


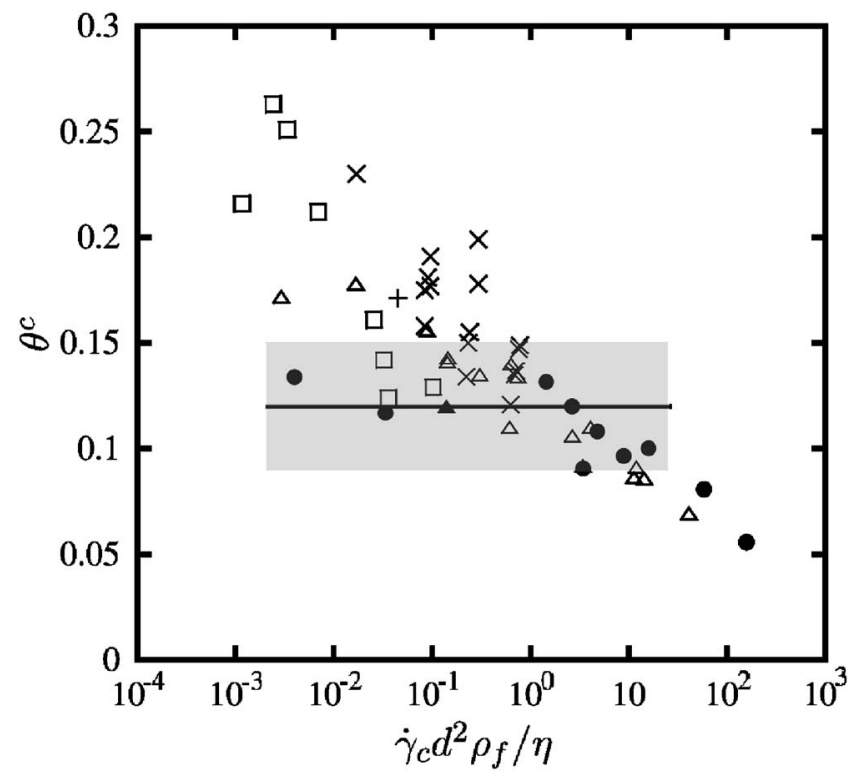

FIG. 5. Critical Shields number $\theta^{c}$ vs $\dot{\gamma}_{c} d^{2} \rho_{f} / \eta$ : experimental data of Charru et al. (Ref. 8) (\), of Loiseleux et al. (Ref. 9) (-), of White (Ref. 6) cited by Loiseleux et al. (Ref. 9) (+), of White (Ref. 10) cited by Mantz (Ref. 11) ( $\square$ ), of Mantz (Ref. 11) (×), and of Yalin and Karahan (Ref. 7) $(\triangle)$. The solid line represents our result with the gray rectangle indicating the error range.

The single datum $(\boldsymbol{\Delta})$ of Charru et $a .^{8}{ }^{8}$ obtained in a Couette channel by measuring the saturated bedload flux is in excellent agreement with our result. There is also a good agreement, within the same range of particle Reynolds number, with the data $(\bullet)$ of Loiseleux et al. ${ }^{9}$ using visual observations of grain motion in a Hele-Shaw cell and defining the erosion threshold as the lowest flow rate for which grains are still being eroded after fifteen minutes. The present method of characterizing the threshold through the cessation of motion yields same threshold value within experimental accuracy than by gradually increasing the flow rate and measuring the saturated particle flux. Indeed, in both experimental procedures, the compaction of the bed has reached a steady state. An important finding of our experiments is that $\theta^{c}$ is found to be constant over the range of small particle Reynolds number explored. This relies on the fact that we have adopted a viscous definition of the shear stress that has been found to be valid in this range. The results of Loiseleux et al. ${ }^{9}$ show a decrease for larger particle Reynolds numbers. They also considered a viscous definition of the shear stress and attributed this decrease to inertial correction to the Stokes drag not accounted by this definition.

The older data $(+, \square, \times, \triangle)$ were mostly obtained using visual observation of the incipient grain motion in open channels. The shear stress was given by delicate measurements of the very low fluid surface gradients. ${ }^{11}$ These data are more scattered and some results present larger values than our result in the range explored. This may be due to (i) their different definition of the shear stress, (ii) the difficulty of the measurement, and (iii) possible unsteadiness of the grain flux. The same order of magnitude is however recovered.

In summary, we have provided reproducible experimental measurements for the onset of grain motion in laminar flow and inferred a critical Shields number for particle erosion that was found to be constant, i.e., $\theta^{c}=0.12 \pm 0.03$, for a wide range of small particle Reynolds number: $1.5 \times 10^{-5}$ $\leqslant \operatorname{Re}_{p} \leqslant 0.76$. We have also shown that adopting a viscous definition of the shear stress is valid in this range.

Support from the Institut Français du Pétrole is gratefully acknowledged by M.O.

${ }^{1}$ J. M. Buffington and D. R. Montgomery, "A systematic analysis of eight decades of incipient motion studies, with special reference to gravelbedded rivers," Water Resour. Res. 33, 1993 (1997).

${ }^{2}$ V. A. Vanoni, "Sediment transportation mechanics: initiation of motion," J. Hydr. Div. 92, 291 (1966).

${ }^{3}$ C. L. Dancey, P. Diplas, A. N. Papanicolaou, and M. Diplas, "Probability of individual grain movement and threshold condition," J. Hydraul. Eng. 128, 12 (2002).

${ }^{4}$ A. S. Paintal, "Concept of critical shear stress in loose boundary open channels," J. Hydraul. Res. 9, 91 (1971).

${ }^{5}$ A. N. Papanicolaou, P. Diplas, N. Evaggelopoulos, and S. Fotopoulos, "Stochastic incipient motion criterion for spheres under various bed packing conditions," J. Hydraul. Eng. 128, 4 (2002).

${ }^{6} \mathrm{C}$. M. White, "The equilibrium of grains on the bed of a stream," Proc. R. Soc. London, Ser. A 174, 322 (1940).

${ }^{7}$ M. S. Yalin and E. Karahan, "Inception of sediment transport," J. Hydr. Div. 105, 1433 (1979).

${ }^{8}$ F. Charru, H. Mouilleron-Arnould, and O. Eiff, "Erosion and deposition of particles on a bed sheared by a viscous flow," J. Fluid Mech. 519, 55 (2004).

${ }^{9}$ T. Loiseleux, P. Gondret, M. Rabaud, and D. Doppler, "Onset of erosion and avalanches for an inclined granular bed sheared by a continuous laminar flow," Phys. Fluids 17, 103304 (2005).

${ }^{10} \mathrm{~S}$. J. White, "Plane bed thresholds of fine grained sediment," Nature 228 , $152(1970)$

${ }^{11}$ P. A. Mantz, "Incipient transport of fine grains and flanks by fluidsextended Shields diagram," J. Hydr. Div. 103, 601 (1977). 